\title{
Nuovi schemi terapeutici nella sclerosi multipla
}

\author{
Maria Pia Amato \\ Professore Associato di Neurologia, Università degli Studi di Firenze
}

\section{Introduzione}

Sono trascorsi più di venti anni dall'introduzione sul mercato dei primi trattamenti "disease modifying" (DMT) per la sclerosi multipla (SM), e da allora diverse nuove terapie hanno raggiunto il mercato e altre sono attualmente in fase avanzata di sperimentazione. È importante sottolineare che la grande maggioranza di questi trattamenti è stata testata nelle forme a decorso recidivante-remittente (RR), su cui pertanto questo contributo si concentrerà.

L'ampliarsi degli scenari terapeutici nelle ultime due decadi ha portato al modificarsi di molti paradigmi. L'elaborazione di nuovi criteri diagnostici che pongono in prima linea nell'algoritmo diagnostico la risonanza magnetica (RM) consente attualmente una

\section{Indirizzo per la corrispondenza:}

Maria Pia Amato

e-mail: mariapia.amato@unifi.it

Accettato: 19/11/2015 - Pubblicato online: 18/12/2015

(C) 2015 The Authors. This article is published by HPS Srl and licensed under Creative Commons Attribution-NC-ND 4.0 International (CC BY-NC-ND 4.0). Any commercial use is not permitted and is subject to Publisher's permissions. Full information is available at www.aboutpharma.com/publishing/riviste/aboutopen/ notevole anticipazione diagnostica e un trattamento in fase precoce di malattia, i cui vantaggi sono indicati da diverse linee di ricerca.

Inoltre, la possibilità di monitorare alla RM l'attività subclinica di malattia consente, insieme al monitoraggio clinico, di identificare tempestivamente $i$ soggetti non-rispondenti o rispondenti subottimali ai DMT, al fine di modificare la strategia terapeutica. I clinici hanno attualmente molte opzioni terapeutiche: farmaci con diverso meccanismo d'azione, diverse modalità di somministrazione (parenterale, orale), diversa efficacia in termini di impatto sull'attività di malattia - terapie di prima, seconda o terza linea a seconda del grado di attività della malattia - e diverso profilo di effetti collaterali ed eventi avversi connessi al loro uso. Questo rende più concretamente attuabile l'obiettivo di una "personalizzazione" della terapia, in base alle caratteristiche cliniche, agli aspetti di RM, ma anche alle caratteristiche demografiche, allo stile di vita e alle preferenze del singolo paziente, mentre si fa sempre più attiva la ricerca di biomarcatori prognostici che possano guidare la scelta terapeutica.

L'approccio graduale a scalare ("escalating") che procede dalle terapie di prima linea a quelle di seconda 
ed eventualmente terza linea in base alla risposta del paziente, viene oggi utilizzato nella maggioranza dei pazienti, ma nei casi aggressivi di malattia si sta affermando sempre più frequentemente un approccio di induzione ("induction"), che utilizzi farmaci più attivi (cui sono connessi maggiori potenziali rischi) fin dall'inizio del trattamento.

Questo capitolo affronterà i progressi connessi all'affermarsi di nuovi schemi terapeutici, destinati in particolare alle forme più attive di malattia, e insieme $\mathrm{i}$ problemi terapeutici ancora aperti e le sfide per la ricerca futura.

\section{Quando iniziare?}

Le molteplici evidenze che attualmente orientano verso il trattamento precoce del paziente con SM sono già state discusse nei contributi precedenti. In sintesi, studi anatomo-patologici e di neuroimmagine hanno documentato come il danno assonale correlato patologico della disabilità neurologica irreversibile - sia un fenomeno già presente nelle fasi precoci della malattia e più rilevante nelle aree di attiva infiammazione e demielinizzazione (danno assonale "acuto"). Nelle fasi più evolute, caratterizzate da un decorso cronico-progressivo, il danno assonale "cronico" sarebbe legato a diversi meccanismi, almeno in parte dissociati dal processo infiammatorio [1]. Inoltre, tutti i farmaci attualmente sul merca- to sono in grado di influenzare la componente infiammatoria del processo patologico, predominante nelle prime fasi di malattia, quando il decorso è RR, mentre al momento non disponiamo di molecole in grado di contrastare efficacemente la neurodegenerazione che predomina nelle fasi progressive. In tale direzione vanno anche i risultati dei trials clinici, che mostrano una maggiore efficacia dei farmaci quando usati in fase precoce di malattia e addirittura al primo episodio clinico, con un'azione meno incisiva $\mathrm{o}$ assente nelle fasi più avanzate e nelle forme progressive, identificando pertanto come "finestra" di opportunità terapeutica ottimale la fase precoce di malattia [1].

È inoltre necessario sottolineare come sia importante iniziare un DMT nei pazienti che hanno la massima possibilità di risposta ottimale, basando la decisione su molteplici fattori - che includono le evidenze di efficacia e sicurezza delle terapie ottenute nei trials clinici e negli studi post-marketing -, sul decorso e attività di malattia del paziente, ma anche sullo stile di vita, le preferenze e l'aderenza alla terapia attesa nel singolo caso (Tabella 1).

I trials di fase III sugli interferoni beta (IFNß-1a e -1b) e su glatiramer acetato (GA) in pazienti con forma RR hanno incluso pazienti che avevano presentato almeno una o due ricadute nell'anno precedente o nei 2 anni precedenti l'ingresso nello studio, e hanno mostrato una riduzione del tasso annualizzato di ricadute e dei parametri di attività alla RM, con va-

Tabella 1. Fattori che influenzano la scelta di inizio della terapia con DMT

\begin{tabular}{|l|}
\hline A favore \\
\hline Sindrome clinicamente isolata ad alto rischio di evoluzione \\
\hline SMRR con almeno una ricaduta nell'anno precedente e/o RM attiva \\
\hline Contro \\
\hline Incertezza diagnostica \\
\hline Programma di gravidanza a breve termine \\
\hline Soggetto ad alto rischio di scarsa aderenza al trattamento \\
\hline $\begin{array}{l}\text { Forma a evoluzione "benigna", in assenza di attività clinica e RM negli ultimi 10-15 anni (necessaria comunque } \\
\text { periodica sorveglianza e rivalutazione clinica e RM) }\end{array}$ \\
\hline
\end{tabular}

DMT, disease modifying therapy; RM, risonanza magnetica; SMRR, sclerosi multipla recidivante-remittente. 
riabili effetti sulla progressione della disabilità [2-15]. La possibilità di confrontare questi trials con quelli condotti sui farmaci orali [fingolimod, dimetilfumarato (DMF), teriflunomide] e sui monoclonali (natalizumab, alemtuzumab) è piuttosto limitata a causa del diverso profilo dei pazienti arruolati che, nei trials più recenti, generalmente si trovano in una fase più precoce di malattia e presentano una minore attività di malattia sia clinica che alla RM rispetto ai trials condotti negli anni 1988-2000 [16]. Nonostante queste considerazioni, è importante notare come alemtuzumab sia stato confrontato nei trials non verso placebo, bensì verso un comparatore attivo, considerato lo "standard terapeutico" e rappresentato da IFNß-1a per via sottocutanea (s.c.) ad alte dosi [17].

\section{Come iniziare?}

Nel momento in cui il clinico decide di iniziare un DMT, deve operare una scelta tra un approccio a scalare ("escalating") o di induzione ("induction") [18].

L'approccio a scalare consiste nell'iniziare con un trattamento di prima linea - inteso generalmente come terapia a efficacia moderata ed elevata sicurez$\mathrm{za}$ - e passare a un trattamento di seconda linea (più efficace ma anche meno sicuro in termini di effetti collaterali e possibili rischi) nel caso di risposta terapeutica insoddisfacente. Questo approccio appare ragionevole per una consistente proporzione di pazienti nella pratica clinica, caratterizzati da attività di malattia lieve o moderata. In alternativa, l'approccio di induzione consiste nell'uso, fin dall'inizio, di un trattamento di seconda linea ad alta efficacia, al fine di ottenere una rapida remissione in una malattia molto attiva, che giustifica l'accettazione da parte del paziente di rischi ed eventi avversi seri [18]. Le problematiche connesse a questo profilo di malattia e approccio terapeutico saranno specificamente trattate nel paragrafo successivo.

Gli IFNß, GA, teriflunomide e DMF sono considerati terapie di prima linea, mentre natalizumab, alemtuzumab e mitoxantrone sono terapie di seconda o terza linea. Fingolimod è approvato come seconda linea in Europa e come prima linea negli Stati Uniti, Canada e altri Paesi. Farmaci immunosoppressori come azatioprina e ciclofosfamide, che non sono registrati per la SM, sono talvolta usati dai clinici come terapie "off-label", rispettivamente come terapie di prima e seconda linea [19].

Gli studi comparativi sulle terapie di prima linea sono limitati. Alcuni studi indicano una maggiore efficacia dell'interferone s.c. ad alte dosi trisettimanale rispetto alla formulazione intramuscolare (i.m.) monosettimanale [20, 21]. L'IFNß ad alte dosi e il GA hanno efficacia simile sui parametri clinici, mentre differiscono lievemente in termini di impatto sui parametri di RM, che è maggiore per l'IFNß, e di profilo di tollerabilità [22-25]. C'è minore esperienza sul DMF, data la sua introduzione recente sul mercato. Uno dei due trials di fase III [14] includeva un braccio trattato con GA. I pazienti trattati con DMF, confrontati al braccio placebo, avevano una maggiore riduzione del tasso annualizzato di ricadute rispetto ai pazienti trattati con GA; anche la riduzione della progressione della disabilità risultava significativa versus placebo solo nel braccio DMF. Lo studio comunque non aveva potenza sufficiente a operare un confronto diretto tra DMF e GA [14]. Negli studi registrativi, teriflunomide mostra un'efficacia simile a quella degli IFNß e del GA, col vantaggio della somministrazione orale [26]. Recentemente, uno studio di piccole dimensioni con azatioprina suggerirebbe una non-inferiorità di questo farmaco rispetto agli IFNß [27], sebbene il dato necessiti di conferme in ampi e rigorosi trials comparativi.

Natalizumab, fingolimod e mitoxantrone sono terapie consolidate di seconda linea. Di più recente introduzione sul mercato l'anticorpo monoclonale alemtuzumab, che in Europa e negli Stati Uniti è stato approvato per le forme "attive" di SM. Tutti i farmaci summenzionati riducono fortemente la frequenza di ricadute rispetto alle terapie standard di prima linea (riduzione di circa il 50\% rispetto all'IFNß) e hanno un profondo effetto sui parametri di attività alla RM [15, 28-30]. Tuttavia, l'effetto sulla progressione della disabilità appare essere meno consistente nei diversi studi. 


\section{Come identificare i pazienti ad alto rischio di evoluzione?}

\section{Forme aggressive: definizione}

La definizione di forma "aggressiva" in letteratura è piuttosto inconsistente, per l'impiego di diversi parametri e criteri di valutazione della disabilità e l'assenza di validi biomarcatori prognostici. Pragmaticamente, si può definire come SM aggressiva la forma in cui si verifichino frequenti e gravi ricadute cliniche con rapido accumulo di disabilità. Talvolta identificata con la SM "maligna", la forma aggressiva è stata definita come il conseguimento di un livello di disabilità pari a 6 sulla Expanded Disability Status Scale (EDSS) entro 5 anni dall'esordio clinico [31]. Uno studio successivo ha distinto tre sottogruppi di forme [32]: il primo caratterizzato dal raggiungimento di EDSS $=6$ entro 5 anni dall'esordio, il secondo dal raggiungimento di EDSS $=6$ entro 40 anni di età e il terzo dall'evoluzione alla forma secondariamente progressiva entro 3 anni dall'esordio. Infine, la definizione di SM aggressiva recentemente utilizzata per descrivere i candidati al trapianto di cellule staminali ematopoietiche [33] includeva il fallimento di terapie convenzionali, frequenti e gravi ricadute cliniche con residua disabilità e attività subclinica alla RM. Si può evidenziare come molte delle definizioni riportate manchino di sensibilità nell'identificare i pazienti a rischio di accumulare disabilità irreversibile nel breve termine. Infatti, il conseguimento di un livello di EDSS pari a 6 rappresenta probabilmente un limite già troppo elevato, oltre il quale il rapporto rischio/beneficio nell'iniziare una terapia di seconda o terza linea può risultare sfavorevole. È stato anche suggerito da un ampio studio di storia naturale [34] che il conseguimento di un livello di EDSS pari a 4 sia già un indicatore di un decorso rapidamente evolutivo nel breve termine e che la prevenzione di ulteriori ricadute, una volta conseguito questo livello di disabilità, non sia più in grado di modificare sostanzialmente l'ulteriore decorso di malattia. Rush et al. [35] suggeriscono pertanto di definire come SM aggressiva le forme di malattia che comportino una o più delle seguenti caratteristiche:

- EDSS = 4 entro 5 anni dall'esordio clinico;

- due o più ricadute con recupero incompleto;
- più di due esami RM che mostrino nuove lesioni in T2 o lesioni gadolinio-positive nonostante il trattamento con DMT;

- mancata risposta a uno o più DMT somministrati per almeno 1 anno.

La Tabella 2 riporta i principali fattori prognostici, derivati da molteplici studi di storia naturale, che possono aiutare il clinico a identificare i pazienti ad alto rischio di progressione.

\section{Forme aggressive: nuovi schemi di trattamento}

Le terapie di prima linea sono in grado di ottenere in molti casi un buon controllo di malattia e studi di follow-up a lungo termine (condotti sugli IFNß e GA) ne documentano una buona sicurezza di impiego [35].

Tuttavia, i pazienti che corrispondono ai criteri di forma aggressiva di malattia sono ad alto rischio di progressione nel breve termine e hanno una limitata "finestra" di opportunità terapeutica.

Le evidenze disponibili suggeriscono che pazienti con forma aggressiva di malattia abbiano una scarsa risposta alle terapie immunomodulanti di prima linea, e in effetti nella storia di questi pazienti si registra in molti casi il fallimento di uno o più farmaci di prima e seconda linea, utilizzati secondo il classico approccio a scalare.

Questa tipologia di pazienti può beneficiare pertanto maggiormente di un approccio di induzione. Particolarmente indicati sono agenti che siano in grado di eliminare le cellule autoreattive, effettrici del processo patologico. Rientrano in questa tipologia di trattamento farmaci come alemtuzumab, mitoxantrone (usato comunque sempre più raramente sia per la presenza di alternative terapeutiche sia a causa del rischio di cardiotossicità e di leucemie) o farmaci immunosoppressivi usati "off-label" come ciclofosfamide per via endovenosa (e.v.), cladribina e lo stesso trapianto di cellule staminali ematopoietiche [35].

Agenti di seconda linea che non siano in grado di eliminare le cellule effettrici, ma solo di tenerle temporaneamente al di fuori del torrente circolatorio, come fingolimod, o di contrastarne l'in- 
Tabella 2. Fattori prognostici sfavorevoli

Fattori demografici

Sesso maschile

Età di esordio $>40$ anni

Etnia africana o latino-americana

\section{Fattori clinici}

Ricadute multifocali

Coinvolgimento piramidale, cerebellare, sfinterico o cognitivo

Recupero incompleto

Necessità di ospedalizzazione per ricaduta

Ricadute frequenti nei primi 3-5 anni di malattia

Breve intervallo tra esordio e prima ricaduta

Rapido accumulo di disabilità (EDSS $\geq 3$ entro i primi 5 anni di malattia)

\section{Aspetti di RM all'esordio}

Elevato carico lesionale in $\mathrm{T} 2$

$\geq 2$ lesioni gadolinio-positive

Presenza di lesioni permanentemente ipointense in T1 ("black holes")

Presenza di evidente atrofia

Lesioni infratentoriali, in particolare midollari

\section{Aspetti di RM al follow-up}

Accumulo di nuove lesioni in T2

Una o più lesioni gadolinio-positive

EDSS, Expanded Disability Status Scale; RM, risonanza magnetica.

gresso nel sistema nervoso centrale (SNC), come natalizumab, possono determinare un controllo efficace dell'attività di malattia, ma in effetti solo temporaneo. La loro sospensione, quando necessaria per diversi motivi, comporta il rischio di un ritorno dell'attività di malattia o addirittura di un "rebound" con incremento dell'attività di malattia oltre i livelli pre-trattamento [36-38].

Tra gli agenti terapeutici sul mercato per il trattamento delle forme attive di SM, particolarmente interessante per l'efficacia elevata e protratta è alemtuzumab, un anticorpo monoclonale umanizzato diretto contro l'antigene CD52 presente sui linfociti $\mathrm{T}$ e B. In ampi trials di fase III alemtuzumab si è dimostrato in grado di ridurre la frequenza di ricadute e la progressione della disabilità sia in pazienti naïve al trattamento, sia in quelli con forma attiva non responsiva alle terapie di prima linea, confrontato con l'IFNß-1a ad alte dosi [17].
Il farmaco viene somministrato alla dose di $12 \mathrm{mg}$ e.v. per 5 giorni, seguiti da un secondo ciclo alla stessa dose per 3 giorni dopo 12 mesi (tale ciclo è ripetibile dopo un ulteriore anno se indicato) e produce una rapida e profonda deplezione linfocitaria, con effetto protratto fino a 1 anno, seguita dal ripopolamento linfocitario e dal "resetting" del sistema immunitario. Oltre alle possibili reazioni infusionali e infezioni opportunistiche, il rischio principale connesso all'uso del farmaco è l'emergenza di disordini autoimmuni (in particolare disordini tiroidei, porpora trombocitopenica, glomerulopatia), anche a distanza dall'ultima dose, il che rende indispensabile un adeguato e protratto follow-up dei pazienti trattati [39]. I dati dello studio di estensione dei trials registrativi documentano che oltre l' $80 \%$ dei pazienti trattati non necessita di terapia durante il terzo anno di follow-up [40, 41] 


\section{Conclusioni}

Il panorama terapeutico nella SM si è evoluto nelle ultime due decadi; tale evoluzione ha portato a nuovi paradigmi terapeutici e nuove sfide cliniche [42]. Il trattamento precoce, il monitoraggio RM per l'identificazione dei non-rispondenti e la tempestiva modifica della strategia terapeutica sono alcuni dei nuovi paradigmi.

Gli obiettivi stessi della terapia si sono riposizionati verso traguardi più ambiziosi, perseguendo l'assenza di attività di malattia, sia clinica (ricadute, progressione della disabilità), sia subclinica alla RM (assenza di nuove lesioni o lesioni captanti gadolinio), in base al nuovo concetto di " $n o$ evidence of disease activity" (NEDA) [43].

Mentre il classico approccio terapeutico a scalare può essere in grado di contenere efficacemente l'attività di malattia nei pazienti con grado di attività lieve o moderato, un approccio induttivo, reso possibile dai nuovi agenti terapeutici sul mercato, può essere indicato per le forme più attive di malattia. L'identificazione precoce di questi pazienti a elevato rischio di progressione è pertanto di importanza critica per un intervento terapeutico più proattivo, in grado di ottenere una rapida e prolungata soppressione dell'attività di malattia, prevenendo lo sviluppo di disabilità irreversibile. Altrettanto critica in questi casi è l'attività di farmacovigilanza, resa possibile anche da Registri del farmaco, attraverso il monitoraggio regolare e il report sistematico degli eventi avversi nel lungo termine.

Altre sfide future riguardano la possibilità di perseguire in modo concreto una personalizzazione della terapia, attraverso l'identificazione di biomarcatori prognostici validi e affidabili da affiancare ai dati clinico-demografici e di RM a oggi disponibili, la terapia delle forme progressive, a oggi prive di strategie terapeutiche efficaci, e il miglioramento delle terapie sintomatiche. Negli ultimi anni, infatti, si è affermata in misura crescente la centralità del percepito e dei valori del paziente, della sua qualità di vita, con una rinno- vata attenzione alla gestione complessiva e multidisciplinare del paziente con SM, che affronti anche il trattamento delle complicanze della malattia e del possibile disagio psico-sociale.

\section{Disclosures}

Conflicts of interest: The author declares she has no conflict of interests related to the article.

\section{Bibliografia}

1. Freedman MS. Induction vs. escalation of therapy for relapsing multiple sclerosis: the evidence. Neurol Sci. 2008;29 Suppl 2:S250-2.

2. The IFNB Multiple Sclerosis Study Group. Interferon beta-1b is effective in relapsing-remitting multiple sclerosis. I. Clinical results of a multicenter, randomized, double-blind, placebo-controlled trial. The IFNB Multiple Sclerosis Study Group Neurology. 1993;43:655-61

3. Paty DW, Li DK. Interferon beta-1b is effective in relapsing-remitting multiple sclerosis. II. MRI analysis results of a multicenter, randomized, double-blind, placebo-controlled trial. UBC MS/MRI Study Group and the IFNB Multiple Sclerosis Study Group. Neurology. 1993;43:662-7.

4. Jacobs LD, Cookfair DL, Rudick RA, et al. Intramuscular interferon beta-1a for disease progression in relapsing multiple sclerosis. The Multiple Sclerosis Collaborative Research Group (MSCRG). Ann Neurol. 1996;39:285-94.

5. PRIMS Study Group. Randomised double-blind placebo-controlled study of interferon beta-1a in relapsing/remitting multiple sclerosis. PRISMS (Prevention of Relapses and Disability by Interferon beta-1a Subcutaneously in Multiple Sclerosis) Study Group. Lancet. 1998;352:1498-504.

6. Johnson KP, Brooks BR, Cohen JA, et al. Copolymer 1 reduces relapse rate and improves disability in relapsing-remitting multiple sclerosis: results of a phase III multicenter, double-blind placebo-controlled trial. The Copolymer 1 Multiple Sclerosis Study Group. Neurology. 1995;45:1268-76.

7. Comi G, Filippi M, Wolinsky JS. European/Canadian multicenter, double-blind, randomized, placebo-controlled study of the effects of glatiramer acetate on magnetic resonance imaging--measured disease activity and burden in patients with relapsing multiple sclerosis. European/Canadian Glatiramer Acetate Study Group. Ann Neurol. 2001;49:290-7.

8. Polman CH, O'Connor PW, Havrdova E, etal.A randomized, placebo-controlled trial of natalizumab for relapsing multiple sclerosis N Engl J Med. 2006;354:899-910.

9. Kappos L NRJN 2010 Unico trovato, ma la sigla del giornale non corrisponde (la sigla NRJN è comunque irreperibile): Kappos L, Radue EW, O'Connor P, et al. A placebo-controlled 
trial of oral fingolimod in relapsing multiple sclerosis. N Engl J Med. 2010;362(5):387-401.

10. Calabresi PA, Radue EW, Goodin D, et al. Safety and efficacy of fingolimod in patients with relapsing-remitting multiple sclerosis (FREEDOMS II): a double-blind, randomised, placebo-controlled, phase 3 trial. Lancet Neurol 2014;13:545-56.

11. O'Connor P, Wolinsky JS, Confavreux C, et al. Randomized trial of oral teriflunomide for relapsing multiple sclerosis. N Engl J Med. 2011;365:1293-303.

12. Confavreux C, O'Connor P, Comi G, et al. Oral teriflunomide for patients with relapsing multiple sclerosis (TOWER): a randomised, double-blind, placebo-controlled, phase 3 trial. Lancet Neurol. 2014;13:247-56

13. Fox RJ, Miller DH, Phillips JT, et al. Placebo-controlled phase 3 study of oral BG-12 or glatiramer in multiple sclerosis. N Engl J Med. 2012;367:1087-97.

14. Gold R, Kappos L, Arnold DL, et al. Placebo-controlled phase 3 study of oral BG-12 for relapsing multiple sclerosis. N Engl J Med. 2012;367:1098-107.

15. Cohen JA, Coles AJ, Arnold DL, et al. Alemtuzumab versus interferon beta $1 \mathrm{a}$ as first-line treatment for patients with relapsing-remitting multiple sclerosis: a randomised controlled phase 3 trial. Lancet. 2012;380:1819-28.

16. Inusah S, Sormani MP, Cofield SS, et al. Assessing changes in relapse rates in multiple sclerosis. Mult Scler. 2010;16:1414-21.

17. Coles AJ, Twyman CL, Arnold DL, et al. Alemtuzumab for patients with relapsing multiple sclerosis after disease-modifying therapy: a randomised controlled phase 3 trial. Lancet. 2012;380:1829-39.

18. Reickman P. Concepts of induction and escalation therapy in multiple sclerosis. J Neurol Sci 2009;277(Suppl 1):S42-5.

19. Sorensen PS. New management algorithms in multiple sclerosis. Curr Opin Neurol. 2014;27:246-59.

20. Durelli L, Verdun E, Barbero P, et al. Every-other-day interferon beta-1b versus once-weekly interferon beta-1a for multiple sclerosis: results of a 2 -year prospective randomised multicentre study (INCOMIN). Lancet. 2002;359:1453-60.

21. Panitch H, Goodin DS, Francis G, et al. Randomized, comparative study of interferon beta-1a treatment regimens in MS: The EVIDENCE Trial. Neurology. 2002;59:1496-506.

22. Mikol DD, Barkhof F, Chang P, et al. Comparison of subcutaneous interferon beta-1a with glatiramer acetate in patients with relapsing multiple sclerosis (the REbif vs Glatiramer Acetate in Relapsing MS Disease [REGARD] study): a multicentre, randomised, parallel, open-label trial. Lancet Neurol. 2008; 7:903-14.

23. Cadavid D, Wolansky LJ, Skurnick J, et al. Efficacy of treatment of MS with IFNbeta-1b or glatiramer acetate by monthly brain MRI in the BECOME study. Neurology. 2009;72:1976-83.

24. O'Connor P, Filippi M, Arnason B, et al. 250 microg or 500 microg interferon beta-1b versus $20 \mathrm{mg}$ glatiramer acetate in relapsing-remitting multiple sclerosis: a prospective, randomised, multicentre study. Lancet Neurol. 2009;8:889-97.

25. La Mantia L, Di Pietrantonj C, Rovaris M, et al. Interferons-beta versus glatiramer acetate for relapsing-remitting multiple sclerosis. Cochrane Database Syst Rev. 2014;7:CD009333.

26. Vermersh P, Czlonkowska A, Grimaldi LM, et al. Teriflunomide versus subcutaneous interferon beta-1a in patients with relapsing multiple sclerosis: a randomised, controlled phase 3 trial. Mult Scler 2014;20:705-16.

27. Massacesi L, Tramacere I, Amoroso S, et al. Azathioprine versus beta interferons for relapsing-remitting multiple sclerosis: a multicentre randomized non-inferiority trial. Plos One 2014;9:e113371.

28. Rudick RA, Stuart WH, Calabresi PA, et al. Natalizumab plus interferon beta-1a for relapsing multiple sclerosis. N Engl J Med. 2006;354:911-23.

29. Cohen JA, Barkhof F, Comi G, et al. Oral fingolimod or intramuscular interferon for relapsing multiple sclerosis. N Engl J Med. 2010;362:402-15

30. Edan G, Comi G, Le Page E, et al. Mitoxantrone prior to interferon beta-1b in aggressive relapsing multiple sclerosis: a 3-year randomised trial. J Neurol Neurosurg Psychiatry. 2011;82:1344-50.

31. Gholipour T, Healy B, Baruch NF, et al. Demographic and clinical characteristics of malignant multiple sclerosis. Neurology. 2011;76:1996-2001

32. Menon S, Shirani A, Zhao Y, et al. Characterising aggressive multiple sclerosis. J Neurol Neurosurg Psychiatry. 2013;84:1192-8.

33. Saccardi R, Freedman MS, Sormani MP, et al. A prospective, randomized, controlled trial of autologous haematopoietic stem cell transplantation for aggressive multiple sclerosis: a position paper. Mult Scler. 2012;18:825-34.

34. Confavreux C, Vukusic S, Adeleine P. Early clinical predictors and progression of irreversible disability in multiple sclerosis: an amnesic process. Brain. 2003;126:770-82.

35. Rush CA, MacLean HJ, Freedman MS. Aggressive multiple sclerosis: proposed definition and treatment algorithm. Nat Rev Neurol. 2015;11:379-89

36. Killestein J, Vennegoor A, Strijbis EM, et al. Natalizumab drug holiday in multiple sclerosis: poorly tolerated. Ann Neurol 2010;68:392-5.

37. O'Connor PW, Goodman A, Kappos L, et al. Disease activity return during natalizumab treatment interruption in patients with multiple sclerosis. Neurology. 2011;76:1858-5.

38. Hakiki B, Portaccio E, Giannini M, et al. Withdrawal of fingolimod treatment for relapsing-remitting multiple sclerosis report of six cases. Mult Scler. 2012;18(11):1636-9.

39. Cossburn M, Pace AA, Jones J, et al. Autoimmune disease after alemtuzumab treatment for multiple sclerosis in a multicenter cohort. Neurology. 2011;77:573-9. 
40. Coles AJ, Arnold DL, Cohen JA, et al. Efficacy and safety of alemtuzumab in treatment-naive patients with relapsing-remitting MS: four-year follow-up of the CARE-MS I study. Mult Scler. 2014;20(1 suppl):P090.

41. Hartung H, Arnold D, Cohen J, et al. Efficacy and safety of alemtuzumab in patients with relapsing-remitting multiple sclerosis who relapsed on prior therapy: four-year follow-up of the CARE-MS II study. Mult Scler. 2014;20(Suppl 1):P043.

42. Gold R, Wolinsky JS, Amato MP, Comi G. Evolving expectations around early management of multiple sclerosis. Ther Adv Neurol Disord. 2010;3:351-67.

43. De Stefano N, Stromillo ML, Giorgio A, et al. Long-term assessment of no evidence of disease activity in relapsing-remitting MS. Neurology. 2015;85(19):1722-3. 